\title{
Resiliencia, Espiritualidad, Aflicción y Tácticas de Resolución de Conflictos en Mujeres Maltratadas
}

\author{
Diva E. Jaramillo-Vélez ${ }^{1}$, Doris E. Ospina-Muñoz ${ }^{2}$, Germán Cabarcas-Iglesias ${ }^{3}$ \\ y Janice Humphreys ${ }^{4}$ \\ ${ }^{1}$ Enfermera. M. Sc. Salud Pública, Facultad de Enfermería, Universidad de Antioquia. \\ E-mail: divajara@catios.udea.edu.co \\ ${ }^{2}$ Psicóloga. M. Sc. Salud Colectiva, Facultad de Enfermería, Universidad de Antioquia. \\ E-mail: doris@tone.udea.edu.co \\ ${ }^{3}$ Licenciado en Matemáticas y Física. M. Sc. Ciencias Matemáticas. Universidad Nacional de \\ Colombia-Sede Medellín. E-mail: gcabarca@unalmed.edu.co \\ ${ }^{4}$ Enfermera y Sicóloga. M. Sc. en Enfermería Pediátrica. Ph. D. en Enfermería. Escuela de \\ Enfermería Universidad de California, San Francisco, USA. \\ E-mail: Janice.humphereys@nursing.ucsf.edu.
}

Recibido 20 Junio 2005/Enviado para Modificación 28 Septiembre 2005/Aceptado Octubre 52005

\section{RESUMEN}

Objetivo Determinar relación de resiliencia y espiritualidad en mujeres maltratadas, con la aflicción, la frecuencia e intensidad del maltrato y la severidad de las lesiones recibidas.

Material y Métodos Muestra de 199 mujeres consultantes en Comisarías de Familia de Medellín, Colombia. Se utilizan escalas de Resiliencia, Perspectiva Espiritual, Listado de síntomas de distrés y Tácticas de conflictos. Se realizó análisis de consistencia interna, correlación y análisis de componentes principales de carácter exploratorio.

Resultados Las escalas mostraron consistencia interna. La resiliencia mostró correlación con la espiritualidad $(r=0.22, p=0.0015)$ y negativamente con el total de síntomas positivos de aflicción (PST) $(r=-0.39, p=<0.0001)$, el índice global de severidad (GSI) $(r=-0.30, p=<0.0001)$ y las 9 dimensiones de síntomas de la SCL-90R.

Conclusiones Los instrumentos utilizados son confiables y validos para medir las variables analizadas. Mujeres con más altos niveles de resiliencia reportaron altos niveles de espiritualidad y menor número de síntomas positivos de aflicción y menos aflicción.

Palabras Clave: Adaptación psicológica, espiritualidad, dolor, conflicto, maltrato conyugal (fuente: DeCS, BIREME). 


\section{ABSTRACT \\ Resilience, spirituality, distress and tactics for battered women's con- flict resolution}

Objective Determining the relationship of resilience and spirituality in battered women to distress, the frequency and intensity of mistreatment and the severity of injury.

Materials and methods A sample was taken of 199 women who consulted Comisarías de Familia de Medellín, Colombia (family police/counselling stations). Resilience scales (RS), spiritual perspective (SPS), SCL-90R and conflict tactics (CTS) were used. Internal consistency, correlation and main exploratory components were measured.

Results The scales revealed internal consistency. Resilience was positively correlated to spirituality $(r=0.22 ; p=0.0015)$ and negatively correlated to total positive distress symptoms (PST) $(r=-0.39 ; p<0.0001)$, the global severity index (GSI) $(r=-0.30 ; p=<0.0001)$ and 9 dimensions of symptoms from SCL90R.

Conclusions The scales used were reliable and valid for measuring the variables being studied. Women with higher levels of resilience reported high levels of spirituality, a lower number of positive distress symptoms and less distress.

Key Words: Resilience, spirituality, conflict resolution, battered women (source: MeSH, NLM).

$\mathrm{M}$

uchas mujeres maltratadas rompen con el maltrato y se recuperan. Sin embargo, no se ha indagado suficientemente sobre los factores que les ayudan a recuperarse ni las relaciones entre ellos. Las investigaciones sobre la influencia de factores protectores en la salud física y mental de las mujeres y los estudios sobre resiliencia en mujeres maltratadas aportan elementos para comprender la adaptación eficaz de estas mujeres y muestran cómo se recuperan del trauma y logran vidas y relaciones personales exitosas (1-4)

McMillen (5) considera que es útil planear estrategias clínicas que ayuden a las personas traumatizadas a transformar los eventos adversos en experiencias de fortalecimiento personal. En este sentido, los estudios en resiliencia se centran en los recursos personales que permiten a las personas enfrentar situaciones adversas (6). En sus inicios, los estudios sobre resiliencia se orientaron a buscar los factores que permitieran una respuesta exitosa a las condiciones estresantes $(7,8)$ pero en la actualidad se concentran en la identificación del juego de tareas o desafíos que están involucrados en el manejo de la crisis personal (9). 
En el campo de la violencia doméstica los estudios sobre resiliencia han encontrado factores que ayudan en la protección y recuperación del maltrato tales como: habilidad para aceptarse y pensar bien de sí, capacidad para hacer atribuciones externas de la culpa, tener locus de control interno, una filosofía positiva de vida y contar con recursos espirituales (10). Estos últimos cumplen una función muy importante en la recuperación del maltrato ya que contribuyen a la disminución del distrés psicológico $(4,11)$. Sin embargo, y a pesar de las recomendaciones de terapistas de familia y de salud mental, la inclusión de la espiritualidad en el tratamiento de la violencia doméstica tiene poco desarrollo en el mundo (12).

Considerando lo anterior, este trabajo pretende conocer los niveles de resiliencia y espiritualidad en las mujeres maltratadas y determinar su relación con distrés, frecuencia e intensidad del maltrato que reciben, así como con severidad de lesiones ocasionadas por el mismo.

\section{MÉTODOS}

Muestra

199 mujeres maltratadas, consultantes, en el 2003, en 11 Comisarías de Familia de Medellín. El número de mujeres seleccionado en cada Comisaría fue proporcional al número de mujeres consultantes en los últimos seis meses. Para la recolección de los datos se visitaron las comisarías en las horas de mayor flujo de público; después de ser atendidas por el comisario(a), a las mujeres se les solicitó participar en el estudio; quienes aceptaban firmaban el consentimiento informado y eran entrevistadas, algunas fueron contactadas telefónicamente.

\section{Instrumentos}

Para medir la resiliencia se utilizó la Escala de resiliencia (RS), elaborada por Wagnild \& Young (13). Ella consta de 25 ítems. Califica, de 1 a 7, el grado de acuerdo o desacuerdo con afirmaciones de dos factores: competencia personal (17 ítems) y aceptación de sí misma y de su vida (8 ítems). La consistencia interna de esta escala, medida con el alpha de Cronbach, fue de 0.94 para toda la escala y 0,9 y 0,8 para cada uno de los factores respectivamente (13).

La espiritualidad se midió con La Escala de Perspectiva Espiritual (SPS), de Pamela G. Reed (14), consta de 10 ítems. En esta, la espiritualidad se refiere a un conocimiento de si mismo, a un sentido de conexión con un ser de 
naturaleza superior o a la existencia de un propósito supremo. El alpha de Cronbach fue, en dos ocasiones, 0,93 (14).

El distrés fue medido con la Lista de Síntomas SCL-90 R, esta identifica nueve dimensiones de síntomas primarios y tres índices globales de distrés: Somatización, Obsesivo-compulsivo, Sensibilidad interpersonal, Depresión, Ansiedad, Hostilidad, Ansiedad fóbica, Ideas paranoides y Psicoticismo. El índice global de severidad (GSI) refleja el nivel actual o profundidad del trastorno. El índice de síntomas positivos de distrés (PSDI) refleja la intensidad de los síntomas. El índice total de síntomas positivos (PST) refleja el número de síntomas reportados positivamente (15).

La frecuencia, intensidad del maltrato y severidad de las lesiones ocasionadas por el mismo, se midieron con La Escala de Tácticas de Conflictos (CTS), de Strauss y Gelles (16). Esta consta de 19 ítems, distribuidos en tres subescalas que miden tipos de resolución de conflictos: forma razonada, agresión verbal y actos de violencia física. Saunders (17) agrega a la CTS una pregunta sobre ataque sexual y cuatro preguntas sobre lesiones físicas y severidad de las lesiones. La confiabilidad de la CTS esta entre 0,88 y 0,95. Su validez se basa en varios estudios que la utilizaron y obtuvieron hallazgos consistentes con investigaciones previas (16).

Análisis

Para cada escala se calculó: la consistencia interna con el Alpha de Cronbach y los promedios; y se realizaron correlaciones entre resiliencia, espiritualidad, índice de gravedad de lesiones de la CTS, los 3 índices globales de distrés y las 9 dimensiones de síntomas de distrés de la SCL-90R. Se realizó análisis de componentes principales exploratorio para observar las relaciones entre los 25 indicadores numéricos utilizados en este estudio.

\section{RESULTADOS}

\section{Características socio demográficas}

La edad de las mujeres fluctuó entre 16 y 72 años, media 35,5 años (DS=10). A pesar de que se entrevistaron mujeres de todos los estratos, el 95\% de las entrevistadas pertenecen al estrato 2. El 84,9\% de las mujeres tienen entre 1 y 25 años de unión marital y el 96,4 \% tiene hijos. El 61,3 \% no esta empleada, de las cuales el $46,5 \%$ son amas de casa, el resto, $14,8 \%$ se reconoce desempleada. 
Dos mujeres no tienen ninguna escolaridad; el 30,6 \% tiene primaria completa o incompleta, el 54,1\% entre secundaria completa o incompleta y el $14,0 \%$ una formación tecnológica.

Aflicción

La consistencia interna de la SCL-90R fue 0,9. Al comparar con valores de referencia de Derogatis para Mujeres No pacientes y pacientes psiquiátricas ambulatorias, las medias obtenidas son significativamente mayores que las de mujeres no pacientes para todas las dimensiones, $\mathrm{p}<0,0001$ y cercanos a los de referencia para pacientes psiquiátricas ambulatorias, superándolos en Somatización, Hostilidad y Paranoia. El 54,2 \% de las mujeres presentaron Síndrome de Estrés Postraumático.

\section{Resiliencia}

La consistencia interna de la escala fue de 0,9. Para el factor de actitud personal fue 0,9 y para las preguntas que se refieren a la aceptación de uno mismo fue 0,7. La media de la RS fue de 147,3, DS= 25, el promedio para la actitud personal fue de $148,7, \mathrm{DS}=18$ y para la aceptación de uno mismo fue de $143,7, \mathrm{DS}=21$.

\section{Tácticas de Conflicto}

Para esta escala la consistencia interna fue 0,8 . El 15,1 \% de las mujeres recibió una combinación de agresiones físicas leves, moderadas, severas y permanentes. La violencia psicológica fue la forma más utilizada, 98,9\%. Las agresiones menores, junto con las agresiones mayores y la violencia sexual, constituyen una combinación frecuente, 78,8 \%. Igualmente, fue alta la utilización en forma conjunta de las agresiones mayores y la violencia sexual, 39,2 \%. Finalmente, la violencia sexual fue utilizada por el 43,2 \% de los compañeros de las mujeres de la muestra.

\section{Espiritualidad}

98,9 \% de las entrevistadas manifestaron tener alguna filiación religiosa: católica u otra religión cristiana. La SPS mostró una consistencia interna de 0,8 . El promedio de la SPS, en una escala de 1-6, fue de 4,8, DS=0,6. La pregunta con mayor media fue: "Mis creencias espirituales son una parte importante de mi vida" 5,4 y DS=0,8. 
Para las mujeres menores de 40 años, el promedio de espiritualidad fue de $4,7, \mathrm{DS}=0,6$ y para las mayores de 40 fue de $5,0, \mathrm{DS}=0,6$, siendo mayor significativamente el promedio en las mayores de 40 años que en las menores $p=0,0011$.

Correlación entre las variables del estudio

Las mujeres con síndrome de distrés postraumático presentaron significativamente un promedio mayor de agresiones mayores con ataque sexual que aquellas que no lo experimentaron. Promedios 14,1 y 8 respectivamente, $\mathrm{p}=0,01$. Igualmente, presentaron significativamente un promedio mayor de agresiones mayores sin ataque sexual, promedios 8,5 y 4,6 respectivamente, $\mathrm{p}=0,05$.

La RS mostró correlación con la SPS $r=0,2, p=0,0015$ y correlación negativa con PST y con GSI. Con el PSDI no se encontró asociación. Las 9 dimensiones de síntomas de la SCL-90R estuvieron significativa e inversamente correlacionadas con el índice de resiliencia en el total de las mujeres estudiadas (Tabla 1).

Tabla 1. Correlación entre resiliencia y aflicción

\begin{tabular}{lll}
\hline \multicolumn{1}{c}{ Índices de Distrés } & \multicolumn{1}{c}{$\mathrm{R}$} & $\mathrm{P}$ valor \\
\hline Índice total de síntomas de distrés (PST) & $-0,388$ & $<0,0001$ \\
Índice de síntomas positivos de distrés (PSDI) & $-0,043$ & 0,5423 \\
Índice global de severidad de síntomas (GSI) & $-0,30313$ & $<0,0001$ \\
Somatización (SOM) & $-0,14204$ & 0,0454 \\
Obsesivo-Compulsivo (O-C) & $-0,34605$ & $<0,0001$ \\
Sensibilidad interpersonal (I-S) & $-0,16532$ & 0,0196 \\
Depresión (DEP) & $-0,37942$ & $<0,0001$ \\
Ansiedad (ANX) & $-0,26262$ & 0,0002 \\
Hostilidad (HOS) & $-0,24651$ & 0,0004 \\
Ansiedad fóbica (PHOB) & $-0,14552$ & 0,0403 \\
Ideación Paranoide (PAR) & $-0,14164$ & 0,04 \\
Psicoticismo (PHY) & $-0,21843$ & 0,0019 \\
\hline
\end{tabular}

Análisis de componentes principales

La relación entre los indicadores utilizados en el estudio (Tabla 2), muestra, en el primer plano factorial (Figura 1), que explica el $57 \%$ de la variabilidad, que el GSI aparece como un buen resumen de todos los indicadores de distrés, mientras el PSDI muestra una menor correlación con estos indicadores. Los índices de violencia y de lesiones son un buen resumen de todos los indicadores de tácticas de solución violenta de conflictos. El único indicador no correlacionado con este conjunto es el índice de resolución de conflictos 
en forma no violenta. Los indicadores de distrés, en forma global, no muestran correlación con los indicadores de uso de la violencia para resolver los conflictos ni con el índice de lesiones. El índice de resiliencia se encuentra correlacionado negativamente con los indicadores de distrés y no correlacionado con los indicadores de violencia. Finalmente, el índice de espiritualidad muestra baja correlación en este plano factorial pero alguna correlación con los indicadores de resiliencia.

$\mathrm{Al}$ relacionar los indicadores numéricos del estudio con grupos ilustrativos de mujeres (Figura 2) según sus características sociodemográficas, se observan cuatro grupos: el primero esta conformado por mujeres del estrato 1 , con secundaria incompleta o primaria incompleta, en unión libre y con edad entre 30 y 39 años; se caracteriza porque tiene puntajes altos en los indicadores de violencia y de distrés y puntajes bajos en resiliencia. El segundo, conformado por mujeres de estrato 2 y 5 , mayores de 60 años, que no trabajan y son separadas, manejan altos niveles de distrés pero no presentan altos puntajes en los indicadores de violencia. El tercer grupo, representa a las mujeres de estratos 3 y 4 con educación tecnológica, universitaria o secundaria completa, que no tienen hijos, casadas y con edades entre 20 y 29 años o de 50 y 59 años, tienen altos puntajes en resiliencia y bajos puntajes en los indicadores de violencia y distrés. El último grupo representa a las mujeres que trabajan y tienen puntajes altos en los indicadores de violencia y en resiliencia.

Figura 1. Análisis global de los 25 indicadores

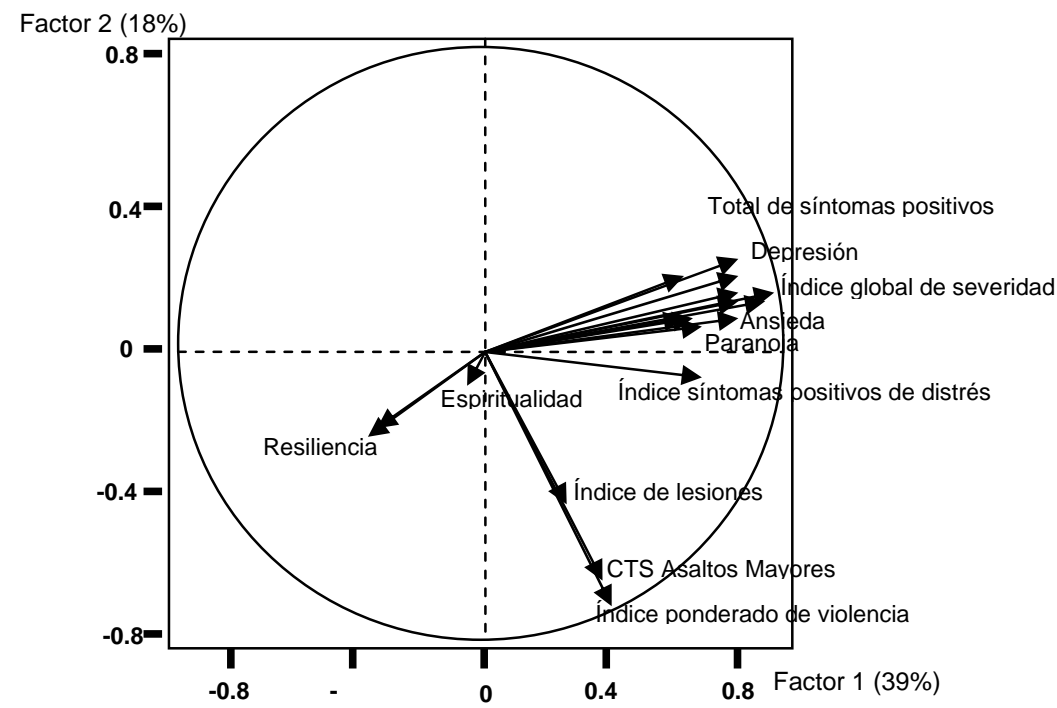


Tabla 2. Resumen de los Indicadores

\begin{tabular}{|c|c|c|c|c|c|}
\hline Indicador & $\begin{array}{c}\text { Número de } \\
\text { Mujeres }\end{array}$ & Media & $\begin{array}{l}\text { Desviación } \\
\text { Estándar }\end{array}$ & Mínimo & Máximo \\
\hline Índice de Espiritualidad & 199 & 4,8 & 0,6 & 2,2 & 6,0 \\
\hline $\begin{array}{l}\text { Índice CTS de resolución no violenta de } \\
\text { conflictos }\end{array}$ & 199 & 1,3 & 1,4 & 0,0 & 6,0 \\
\hline Índice CTS Verbal & 199 & 4,3 & 1,2 & 0,1 & 6,0 \\
\hline Índice CTS Violencia & 199 & 1,7 & 1,3 & 0,0 & 5,5 \\
\hline Índice CTS Asaltos Menores & 199 & 37,5 & 37,8 & 0,0 & 125,0 \\
\hline Índice CTS Asaltos Mayores & 199 & 11,3 & 18,2 & 0,0 & 100,0 \\
\hline $\begin{array}{l}\text { Índice CTS Asaltos Mayores sin Asaltos } \\
\text { sexuales }\end{array}$ & 199 & 6,6 & 14,1 & 0,0 & 100,0 \\
\hline Índice Ponderado de Violencia & 199 & 48,9 & 52,0 & 0,0 & 225,0 \\
\hline Índice de Lesiones & 199 & 10,3 & 16,8 & 0,0 & 108,0 \\
\hline Total de Síntomas Positivos (PST) & 199 & 47,3 & 17,3 & 6,0 & 84,0 \\
\hline Índice Global de Severidad (GSI) & 199 & 1,2 & 0,6 & 0,1 & 2,7 \\
\hline $\begin{array}{l}\text { Índice de Síntomas Positivos de Distrés } \\
\text { (PSDI) }\end{array}$ & 199 & 2,3 & 0,5 & 1,0 & 3,7 \\
\hline Somatización (SOM) & 199 & 1,3 & 0,8 & 0,0 & 3,7 \\
\hline Obsesivo - Compulsivo (O-C) & 199 & 1,4 & 0,7 & 0,0 & 3,5 \\
\hline Sensibilidad Interpersonal (S I) & 199 & 1,3 & 0,7 & 0,0 & 3,2 \\
\hline Depresión (DEP) & 199 & 1,7 & 0,8 & 0,0 & 3,6 \\
\hline Ansiedad (ANX) & 199 & 1,1 & 0,8 & 0,0 & 3,4 \\
\hline Hostilidad (HOS) & 199 & 1,2 & 0,9 & 0,0 & 4,0 \\
\hline Ansiedad Fóbica (PHOB) & 199 & 0,7 & 0,7 & 0,0 & 3,2 \\
\hline Ideación Paranoide (PAR) & 199 & 1,4 & 0,8 & 0,0 & 4,0 \\
\hline Psicoticismo (PSY) & 199 & 0,9 & 0,7 & 0,0 & 2,7 \\
\hline Síntomas de Estrés Postraumático & 199 & 1,1 & 0,7 & 0,0 & 2,7 \\
\hline Índice de Resiliencia & 199 & 147,3 & 17,9 & 90,0 & 175,0 \\
\hline Resiliencia Actitud Personal & 199 & 5,9 & 0,7 & 3,4 & 7,0 \\
\hline Resiliencia Aceptación de uno mismo & 199 & 5,7 & 0,8 & 3,5 & 7,0 \\
\hline
\end{tabular}

\section{DISCUSIÓN}

Las mujeres del estudio presentan altos niveles de distrés y altos niveles de violencia. La relación entre estos factores es compleja, ya que el estrés social puede ser causa de violencia intrafamiliar como la violencia intrafamiliar ser causa de estrés $(4,18-21)$. La relación entre PTSD y severidad de las lesiones es clara; las mujeres que presentaron síndrome de estrés postraumático habían sido sometidas, de manera más frecuente que las demás, a lesiones severas y moderadas acompañadas de violencia sexual, lo cual es reportado en otros trabajos sobre el tema $(4,22,23)$. 
Figura 2. Indicadores y Variables Sociodemográficas

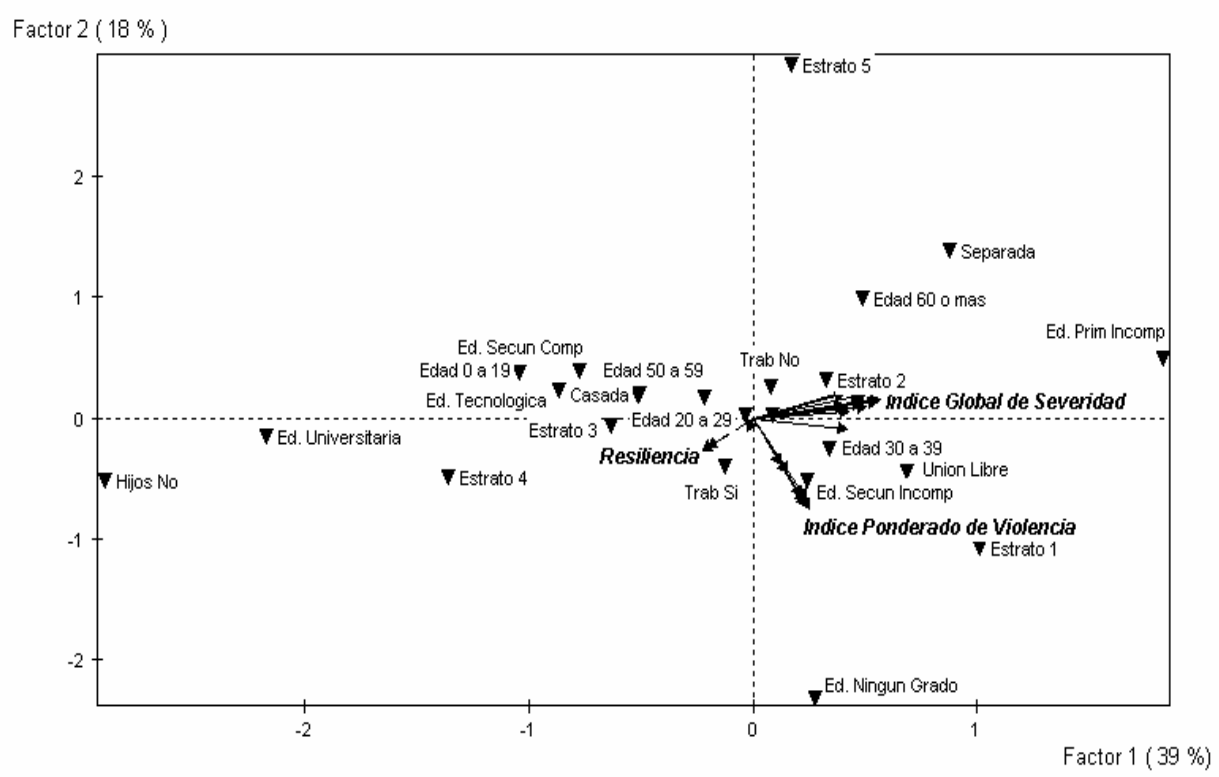

La predominancia del uso de la violencia psicológica por parte del compañero de las mujeres de este estudio, concuerda con resultados de estudios previos, en los que se afirma, además, que este tipo de violencia es la menos denunciada, la más difícil de detectar y la más difícil de afrontar; causando graves problemas emocionales y de salud a las mujeres $(22,24,25)$. Los resultados con respecto al distrés y la violencia corroboran la difícil situación que experimentan las mujeres maltratadas (26). De ahí que pueda hacerse un llamado a considerar ambos factores cuando se realizan intervenciones con mujeres maltratadas.

En este estudio al igual que en otros que miden la violencia doméstica $(27,28)$, no se observa asociación estadísticamente significativa de esta violencia con factores sociodemográficos. Sin embargo, llama la atención el hecho de que las mujeres que no trabajan, tienen bajo nivel de escolaridad y bajos ingresos aparecen como las más vulnerables al presentar altos niveles de violencia, de distrés y baja resiliencia. Por el contrario, aquellas mujeres de estrato 3 y 4 con mayores niveles educativos, tienen altos niveles de resiliencia y bajos de violencia y distrés. Son importantes los hallazgos con respecto a la resiliencia y la espiritualidad y la relación de la primera con la reducción del distrés. Los datos muestran que la resiliencia parece contribuir 
de manera importante a la reducción de la profundidad del distrés y al número de síntomas reportados. Los altos niveles de resiliencia y espiritualidad hallados sugieren la posibilidad de implementar intervenciones en las que se exploren los recursos personales y sociales que coadyuvan a la superación de la experiencia adversa. En este sentido, futuros estudios que indaguen sobre la manera de promover la resiliencia y la espiritualidad, considerados como factores que contribuyen al autocuidado y la adaptación exitosa $(2,4,10,11,29-33)$, pueden aportar en la transformación de las intervenciones con mujeres maltratadas. Esta transformación se hace urgente ya que los últimos estudios en nuestro medio $(34,35)$ muestran que el problema es creciente y que los hombres muestran una tendencia a adoptar comportamientos violentos como forma de resolución de conflicto, aún desde temprana edad $(36,37)$, lo cual deriva en graves consecuencias para la salud de las mujeres.

Siguiendo la definición de resiliencia planteada por Rutter (30) y enfoque de resiliencia del instrumento que se utilizó en este estudio (11), puede afirmarse que las mujeres de la muestra presentan características de personalidad que pueden conducir a las mujeres maltratadas, bajo adecuada orientación, a convertirse en personas resilientes. Lo mismo puede afirmarse con respecto a los altos niveles de espiritualidad, asociados a la resiliencia. Los estudios que miden la violencia doméstica hacen énfasis en las características del agresor o de la víctima, logrando en la mayoría de los casos descripciones de los factores negativos o patológicos $(26,38)$ sin completarse con la descripción de factores positivos de personalidad que enriquezcan la mirada sobre el problema. En este sentido, se sugiere la utilización de escalas de tal manera que se pueda incrementar la descripción de las mujeres y las situaciones que enfrentan

Agradecimientos. Investigación realizada por el grupo de investigación Salud de las Mujeres, Facultad de Enfermería, Universidad de Antioquia, con financiación de la Universidad de Antioquia y COLCIENCIAS.

\section{REFERENCIAS}

1. Humphreys J. Resilience in sheltered battered women. Issues mental health nurs 2003; 24: 137-152.

2. Humphreys J. Turning and adaptations in resilient daughters of battered women. J Nurs Scholarsh 2001; 33(3):245-251.

3. Humphreys J. Growing Up in a violent home: the lived experience of daughters of battered women. J Fam Nurs 2001; 7(3): 244-260. 
4. Humphreys J. Spirituality and Distress in sheltered Battered Women. J Nurs Scholarsh 2000; 32(3): 273-278.

5. MacMillan JC. Better for it: How people benefit from adversity. Soc. work 1999; 44: 455-467.

6. Munist M, Santos H, Kotlirenco MA. En: Infante F, Grotberg E. Manual de identificación y promoción de la resiliencia en niños y adolescentes [Internet]. Organización Panamericana de la Salud. Disponible en: http://www.paho.org/spanish/hppadol.htm. Consultado 15 noviembre 2003.

7. Masten A, Best K. Garmezy, N. Resilience and development: Contributions from the study of children who overcome adversity. Dev Psychopathol 1990; 2: 425-444.

8. Polk L. Toward a middle-range theory of resilience. Adv Nurs Sci 1997; 19(3):113

9. Rutter M. Psychosocial resilience and protective mechanisms. In: Rolf J, Masten AS, Cicchetti D, Nuechterlein KH, Weintraub S, eds. Risk and protective factors in the development of psychopatholgy. New York: Cambridge University Press; 1990. p. 181-214.

10. Valentine LN, Feinauer L.L. Resilience factors associated with female survivors of childhood sexual abuse. Am J Fam Ther 1993; 21(3): 216- 224.

11. Wagnild G, Young HM. Resilience among older women. J Nurs Scholarsh 1990; 22(4): 252-255.

12. Senter K, Caldwell K. Spirituality and the maintenance of change: a phenomenological study of women who leave abusive relationships. Contemp. fam. ther 2002; 24(4): 543-563.

13. Wagnild G, Young HM. Development and psychometric evaluation of the Resilience Scale. J Nurs Meas 1993; 1:165-177.

14. Reed PG. Developmental resources and depression in the elderly. Nurs Res 1986; 35: 368-374.

15. Derogatis LR. Symptom checklist-90-R. Administration, scoring, and procedures manual. Minneapolis, MN: National Computer Systems; 1994.

16. Straus MA, Gelles RJ. Societal change and change in family violence from 1975 to 1985 as revealers by two national surveys. J Marriage Fam 1986; 48: 465-479.

17. Saunders DG. Posttraumatic stress symptom profiles of battered women: A comparison of survivors in two settings. Violence Vict 1994; 9: 31-44.

18. McCubbin HI, Joy CB, Cauble AE, Comeau JK, Patterson JM, Needle RH. Family Stress and coping a decade review. J Marriage Fam 1980; 42:855871.

19. Gelles R. Violence in the family: A review of research in the seventies J Marriage Fam 1980; 42:873-885.

20. Finn J. The Stresses and coping behavior of battered women In: Soc Casework 1985; 66(6): 341-349.

21. Dienemann J, Boyle E, Baker D, Resnick W, Wiederhorn N, Campbell J. Intimate Partner Abuse Among Women Diagnosed With Depression. Issues Ment Health Nurs 2000; 21(5):499-513. 
22. Hilberman. E. Overview: “The Wife-Beater's Wife” Reconsidered. Am J Psychiatry 1980; 137:1336-1346 .

23. Astin MC, Ogland SN, Foy DW, Coleman EM. Post-Traumatic stress disorder and childhood abuse in battered women. J Consult Clin Psychol 1995; 63(2):308-312.

24. Bennett H, Cohen R, Ellard JH. Coping with an abusive relationship: How and why do women stay? J Marriage Fam 1991:311-325.

25. Culay A, Santana F, Rodríguez R, Pérez C. Mujer y violencia ¿Un problema de salud Comunitario?. Rev. Cubana Med Gen Integr. 2000; 16(5):450-454.

26 Organización Panamericana de la Salud. Informe mundial sobre la violencia y la salud: Publicación científica 2003; 588: 97-110.

27. Klevens J. Violencia contra la mujer. Factores de riesgo y medidas de prevención. Serie criterios de justicia 1998; 9:4-14.

28. Jaramillo DE, Uribe TM. Violencia doméstica en Medellín (Colombia). Un problema que afecta la salud de las mujeres. Index enferm 2000; 30:17-21.

29. Garmezy N. A closing note: Reflections on the future. In: Rolf J, Masten A, Cicchetti D, Nuechterlein K, Weintraub S, eds. Risk and protective factors in the development of psychopathology. New York: Cambridge University Press; 1990. p. 527-534.

30. Rutter M. Psychosocial resilience and protective mechanisms. In: Rolf J, Masten AS, Cicchetti D, Nuechterlein KH, Weintraub S, eds. Risk and protective factors in the development of psychopatholgy. New York: Cambridge University Press; 1990. p. 181-214.

31. Watt NF, David JP, Ladd KL, Shamos S. the life course of psychological resilience: A phenomenological perspective on deflecting life's slings and arrows. J Prim Prev 1995; 15:209-245.

32. Werner E, Smith R. Overcoming the odds high risk children from birth two adulthood. New York: Cornell University Press; 1993.

33. Rackin JM. The roles of sense of coherence, spirituality, and religion in responses to trauma. Doctoral dissertation, California School of professional Psychology, 1999 Dissertation Abstracts International 1999; 59, 5106.

34. República de Colombia, Profamilia. La violencia y los derechos humanos en la mujer, salud sexual y reproductiva en Colombia. Encuesta Nacional de Demografía y Salud. Bogotá: Printex Impresores; 1995.

35. República de Colombia, Profamilia. Violencia contra las mujeres y los niños. Salud sexual y reproductiva en Colombia. Encuesta Nacional de Demografía y Salud. Bogotá: Printex Impresores; 2000.

36. Perez I, Pinzón AM, González R, Sánchez J. Influencia de la televisión violenta en niños de una escuela pública de Bogotá, Colombia. Rev. Salud pública 2005; 7(1):70-88.

37. Amortegui D. Violencia en el ámbito universitario: el caso de la Universidad Nacional de Colombia. Rev. Salud pública 2005; 7(2):157-165.

38. Fajardo JP, Fernández K, Escobar OB, Colombia. Estilo de vida, perfil psicológico y demográfico de mujeres maltratadas por su cónyuge [Internet].Disponible en: http://www.psicologiacientifica.com. Consultado 20 de noviembre de 2003. 